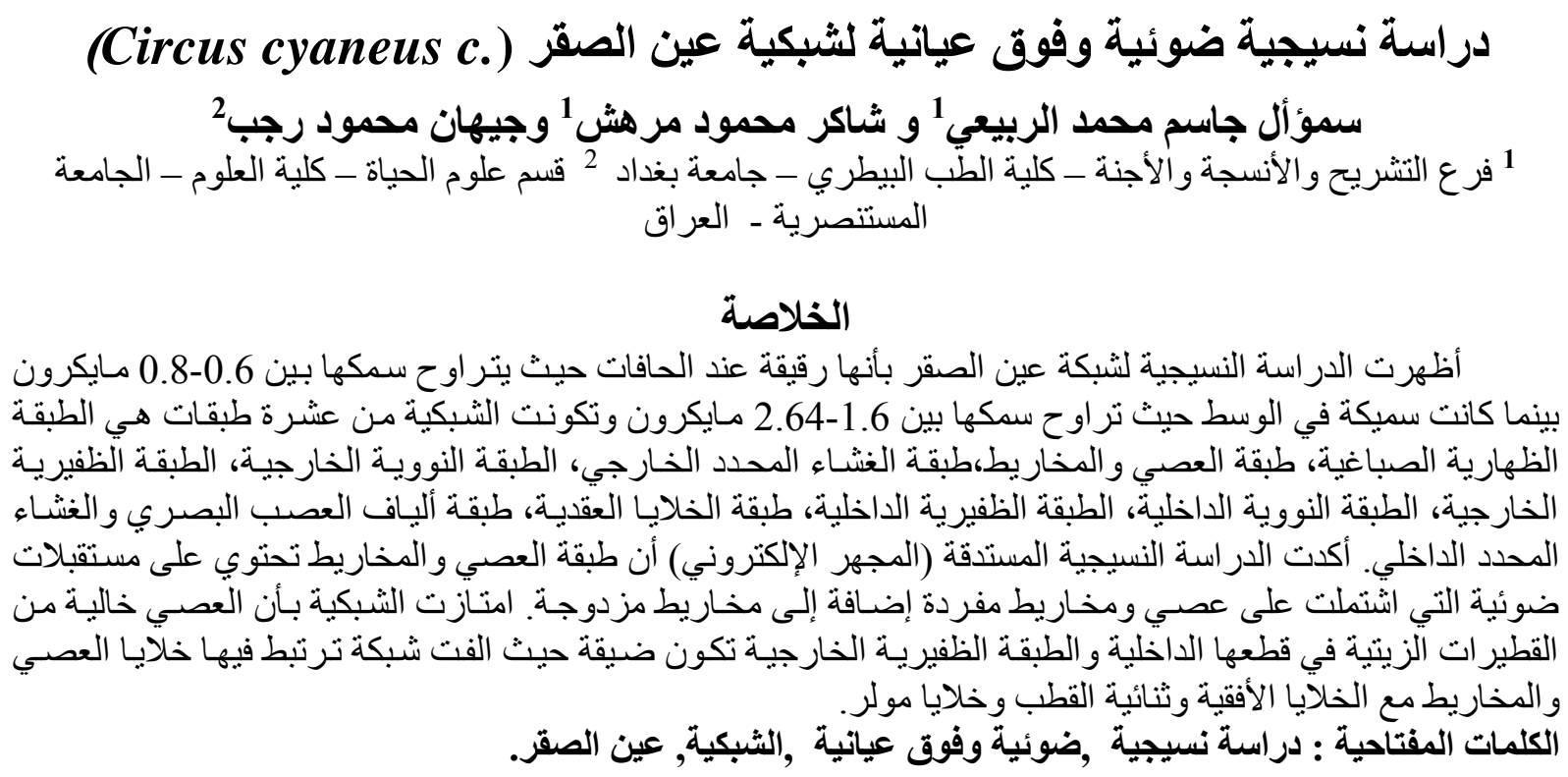

\title{
Histological Studies on the Retina of the Falcon "S Eye Ball (Circus Cyaneus $C$.) Under Light and Electron Microscopy
}

\section{Samawal Jassim Mohamed Al-Robaae ${ }^{1}$, Shakir Mahmood Mirhish ${ }^{1}$ and Jehan Mahmood Rajab ${ }^{2}$}

${ }^{1}$ Department of Anatomy, histology and embryology, College of Veterinary Medicine, University of Baghdad. ${ }^{2}$ Department of Biology, College of Sciences, Al-Mustansiriyah University, Iraq

Accepted: 7 /2/2012

\section{Summary}

The histological study showed that the retina of falcon"s eyeball was thin at the periphery and ranges between $0.6-0.8 \mu$ but it was thick at center 1.68-2.64 $\mu$. The retina consists from ten layers: pigmented epithelium, rods and cons layer, external nuclear layer, external plexiform layer, internal nuclear layer, internal plexiform layer, Gangilionic cells layer, neurofibres layer and internal limiting membrane layer. The ultra-structural study stated that the rods and cons layers contained single rods with single and double cons. The retina characterized by lacking of the oil droplets in the internal segments of rods with narrow external plexiform layer in order to form network connecting rods and cones, horizontal cells, bipolar cells and Muller's cells.

Keywords: Histological Studies, Retina, Falcon Eye Ball, Circus Cyaneus , Light and Electron Microscopy.

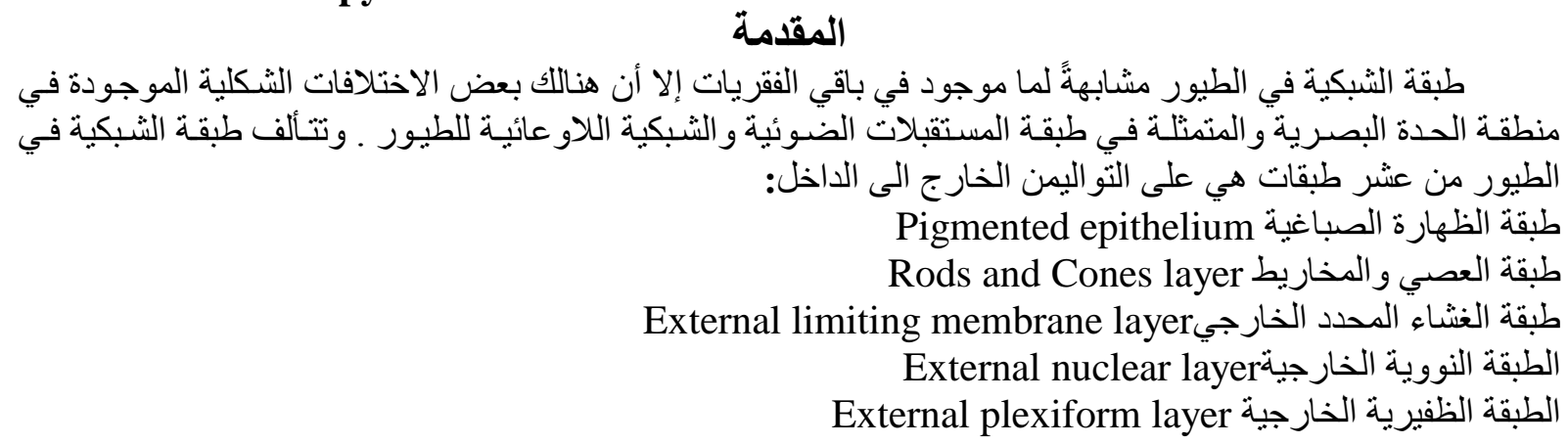


الطبقة النووية الداخلية Internal nuclear layer

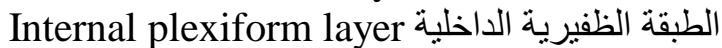

طبقة الخلايا العقدية Ganglionic cells layer

مeuro fibrous layerreبقة الألياف العصبياء

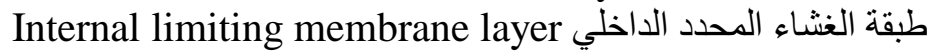

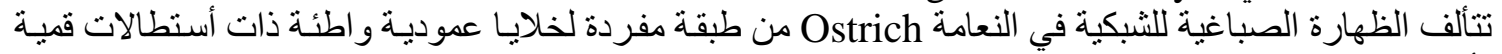

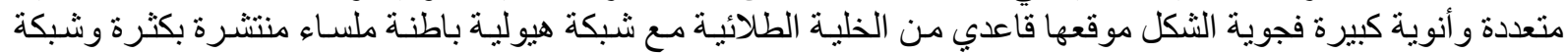

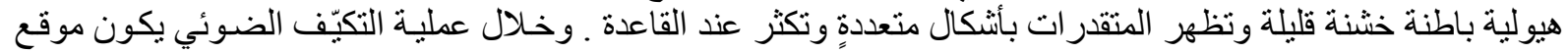

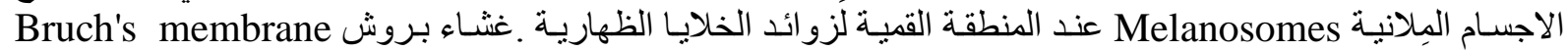

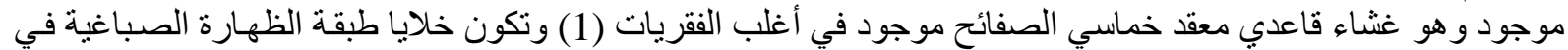

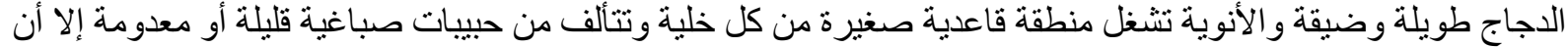

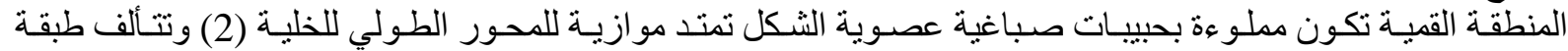

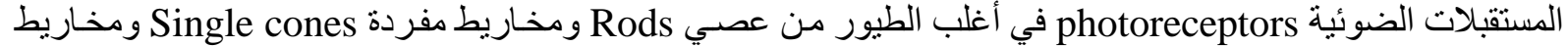
مزدوجة Double cones إلا أن نسبها نختلف من نوع لأخر ففي طير البانشق أحمر الذيل (Buteo jamaicens) تكون

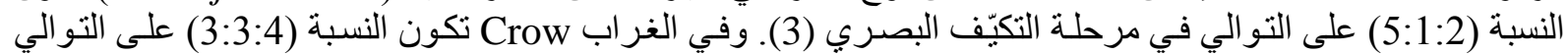

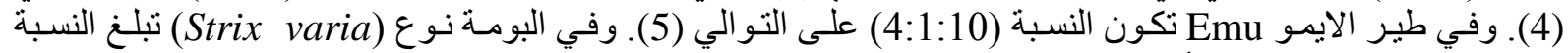

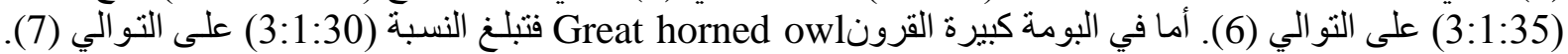

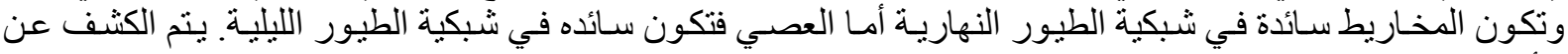

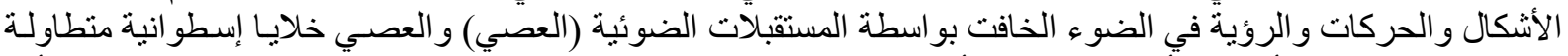

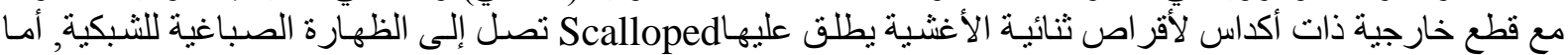

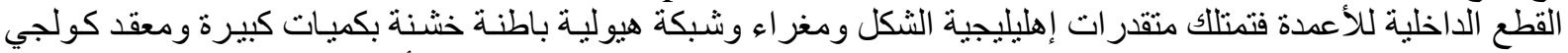

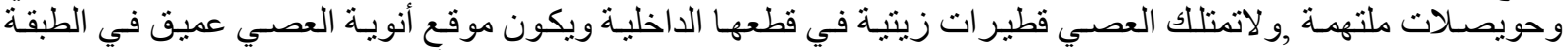

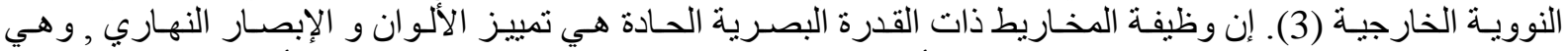

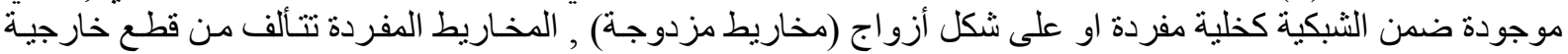

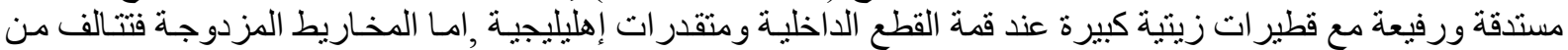

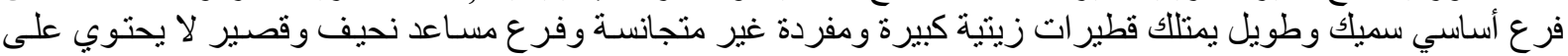

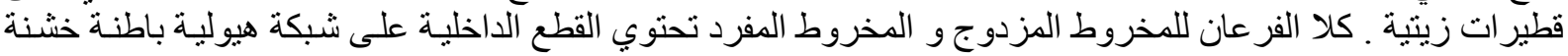

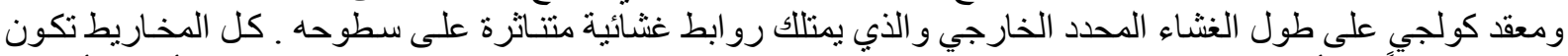

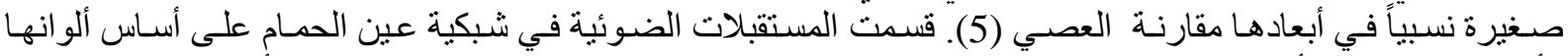

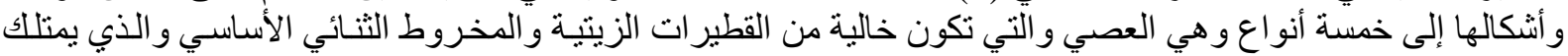

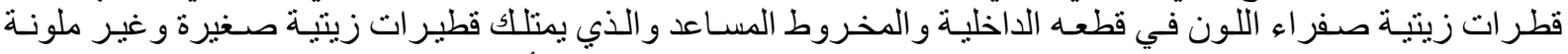

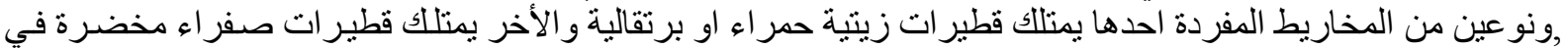

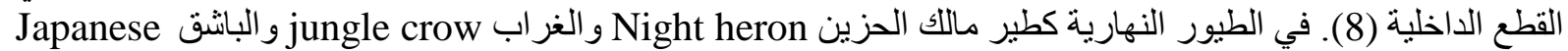
lesser sparrow hawk

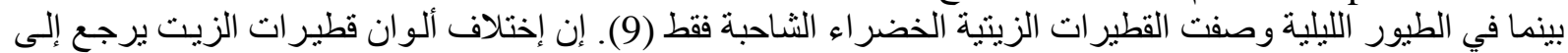

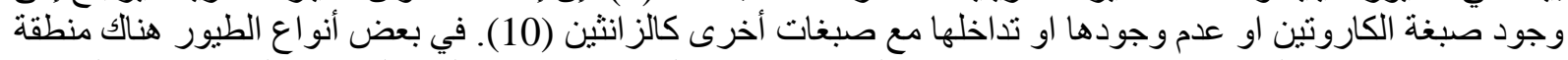

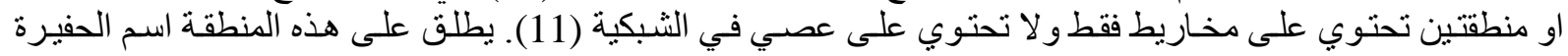

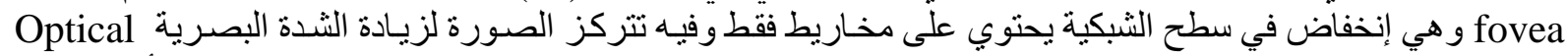
جئitensity جميع الأنو اعوصفت الحفيرة الأنفية Nasal fovea وماعدا البو اشق Hawks و النسور Temporal fovea

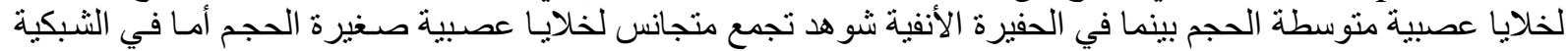

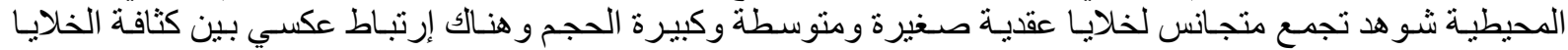
العقدية وحجم أجسام الخلايا في جميع الأنواع المدروسة (11).

\section{المواد وطرائق العمل}

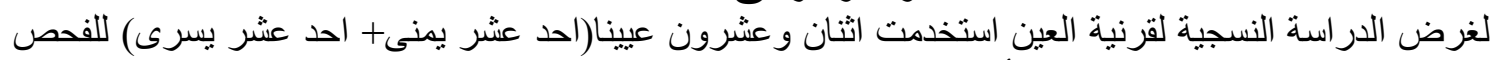

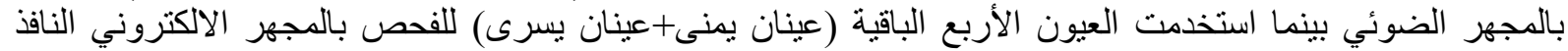
Transmission electron microscope تر اوحت أوزان الطيور بين 1400-850 غم وأعمار ها تراوحت بين 22-14 شهراً. 
وضعت العينات في محلول الفورمالين 10\% لمدة 72 ساعة لغرض تلثنيت العينات

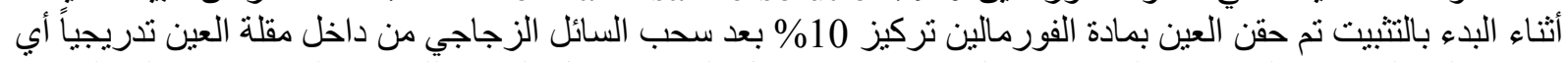

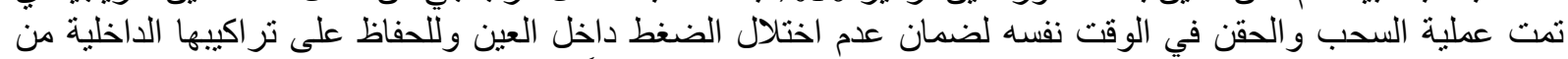

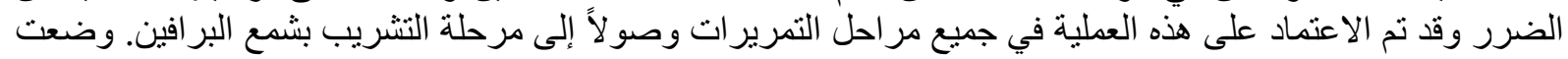

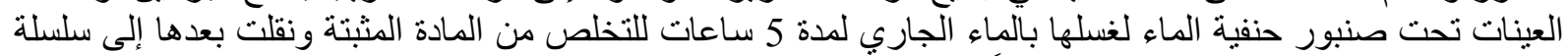

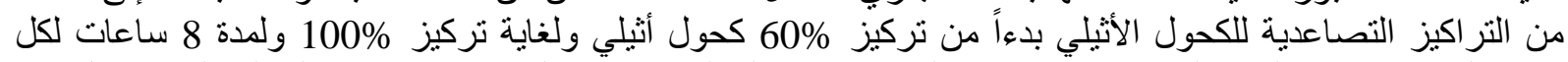

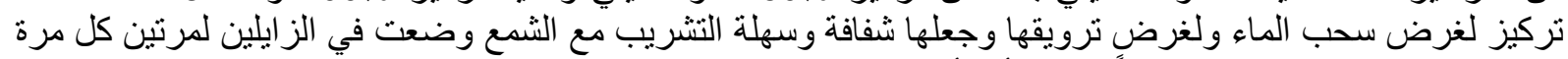

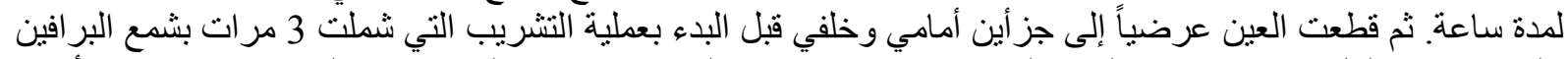

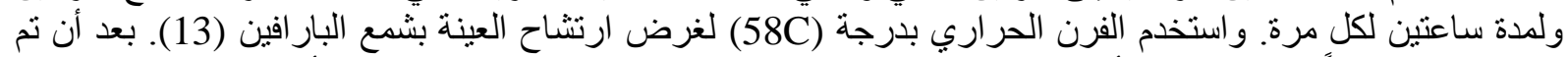

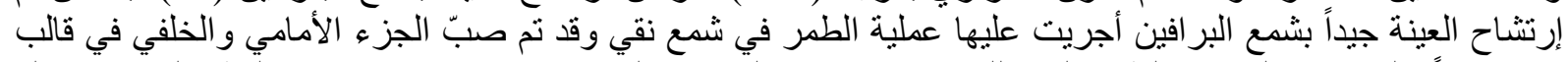

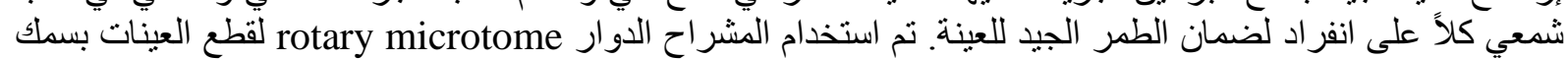

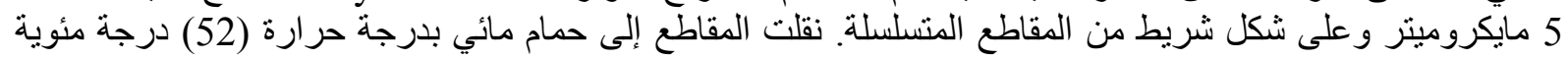

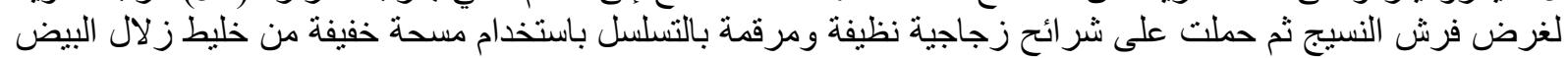

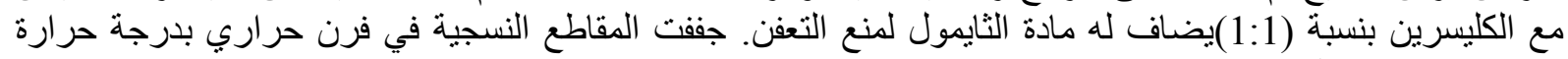

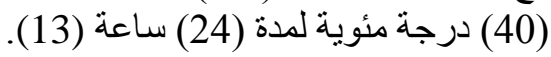

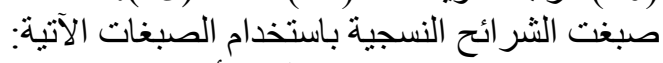

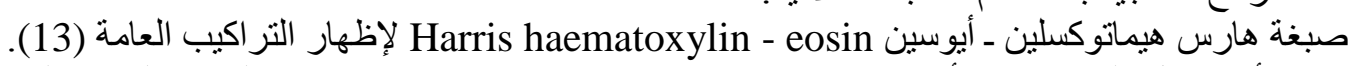

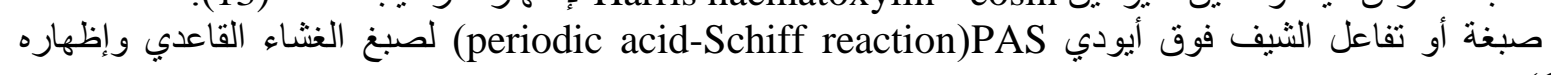

صبغة الماسون ثلاثية الكروم masson trichrome method لإظهار ألياف النسيج الضام و الخلايا بشكل عام (15).

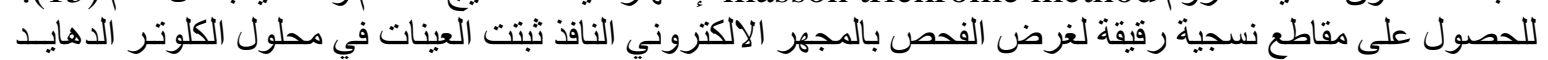
بlutrealdehyde

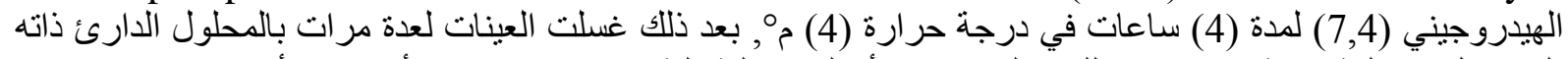

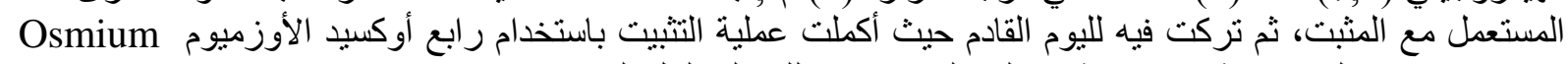
1\% tetroxide

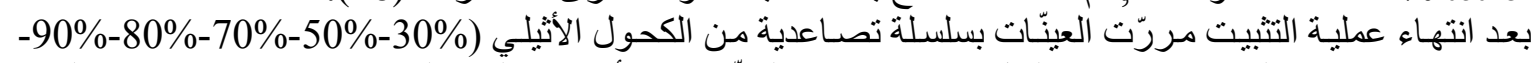

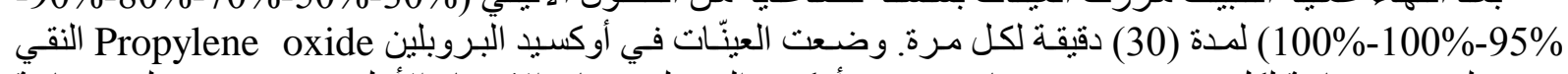

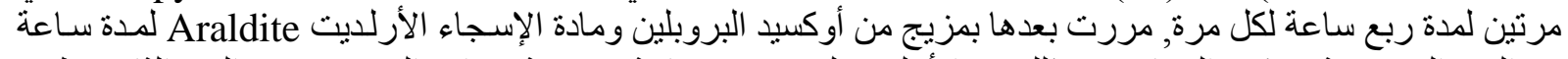

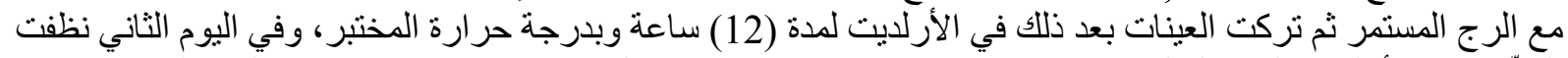

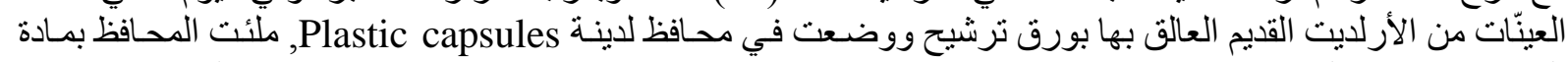

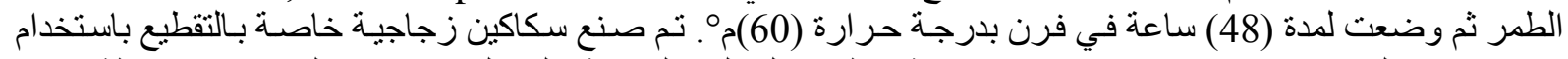

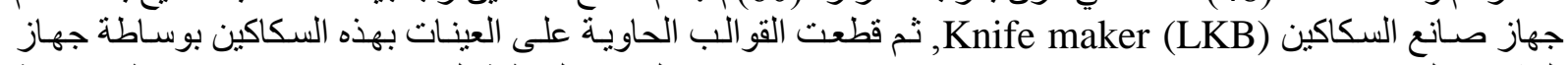

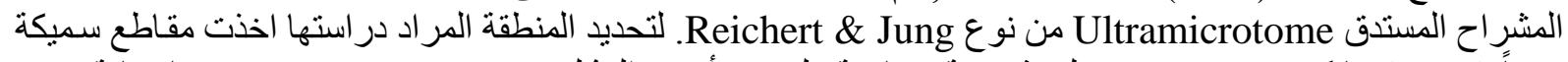

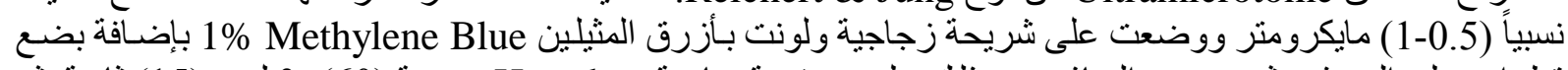

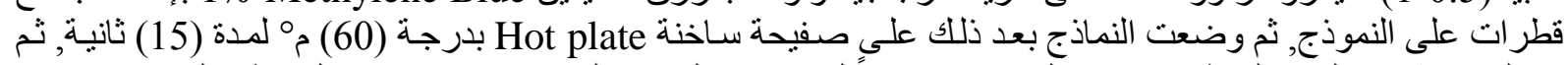

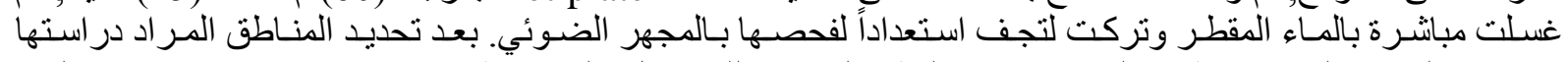

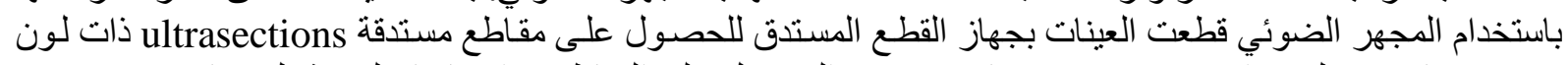

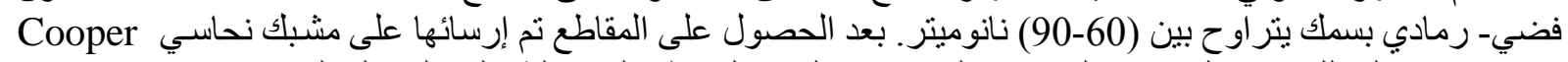

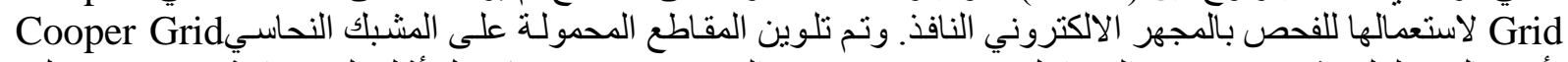

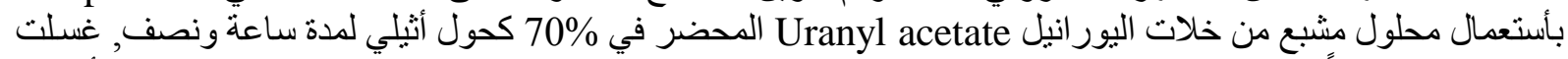

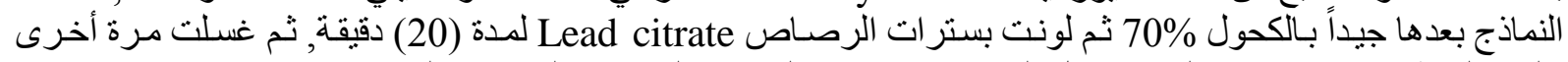

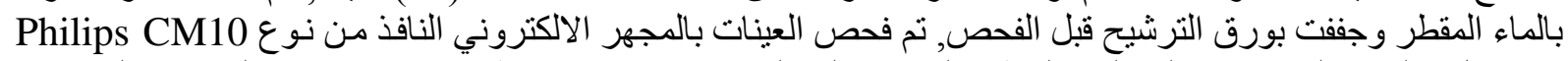

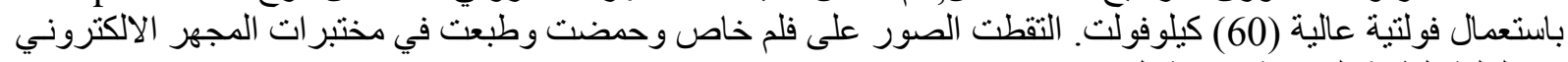
في الكلية الطبية العر اقية/جامعة النهرين.

\section{النتائج والمناقشة إنشي}

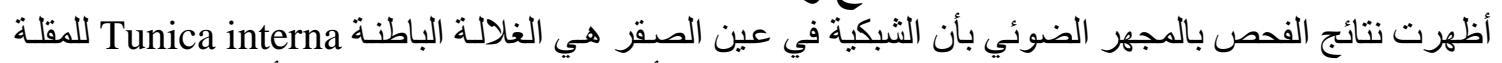

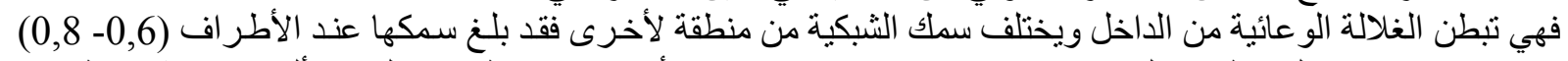

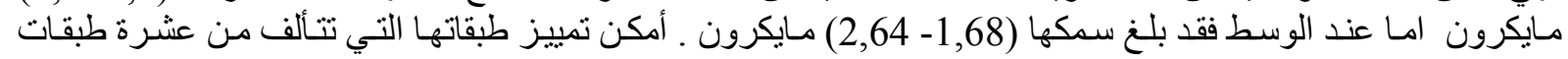
و اضحة ومميزة و هي كما في اللبائن(17) على التو الي من الخارج الى الداخل :- 
طبقة الظهارة الصباغية Pigmented epithelial layer

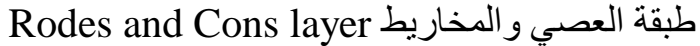

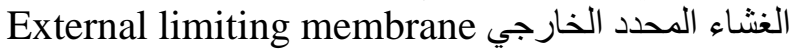

الطبقة النووية الخارجية Outer nuclear layer الطية الخية

Outer plexiform layer الطبقة الظفيرية الخارجية

الطبقة النووية الداخلية الطية الطية

الطبقة الظفيرية الداخلية Inner plexiform layer

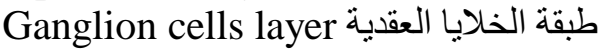

Oبقة الياف العصب البصري Optic nerve fibers layer

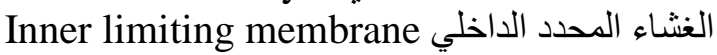

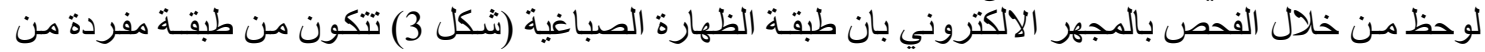

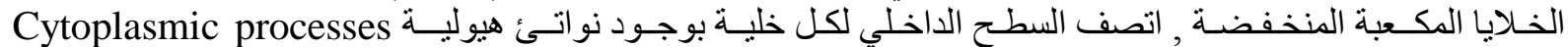
تحيط بسطوح القطع الخارجية للمستقبلات الضوئية Photoreceptors كذلك تكثر أعداد المتقدرات Mitochondria في التي

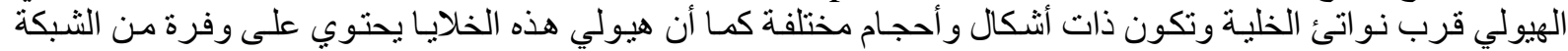

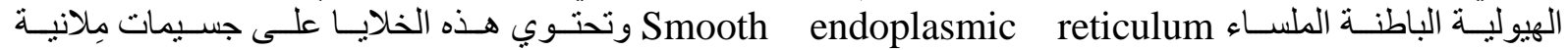

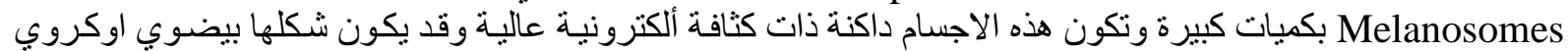

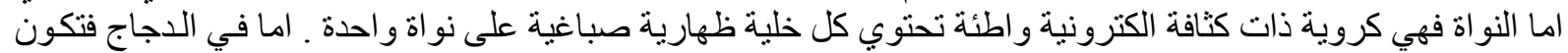

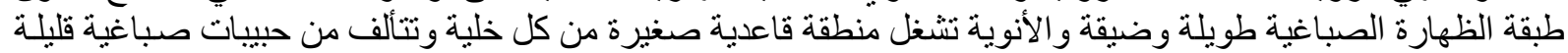

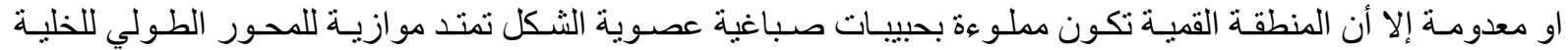

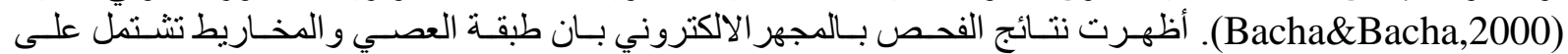

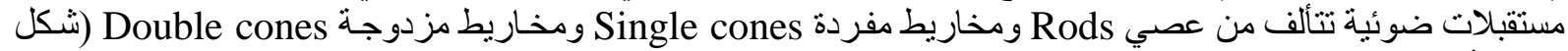
4) ـ تتألف العصي من قطع خارجية Outer segments ذات إرتباط طليق او سـائب مـع النو اتئ التي تبرز من السطح

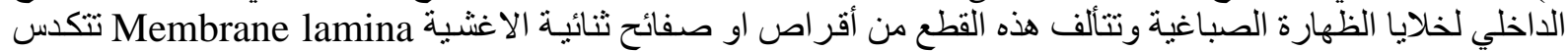

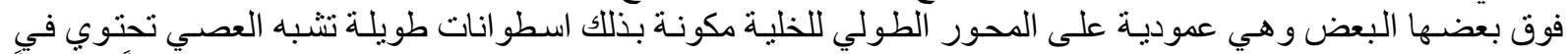

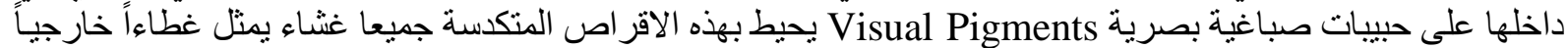

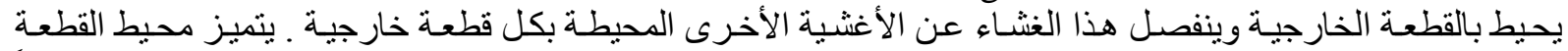

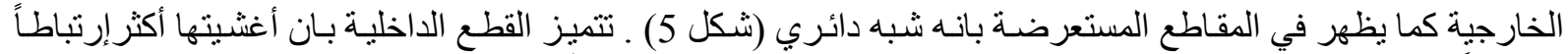

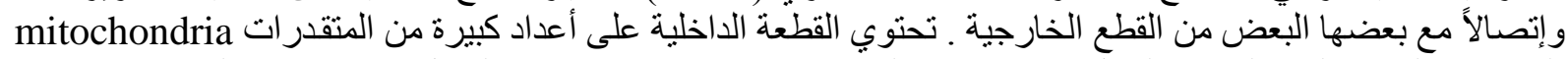

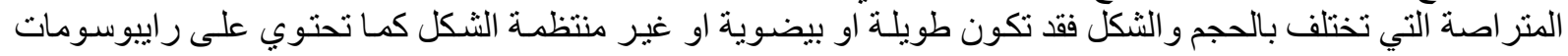

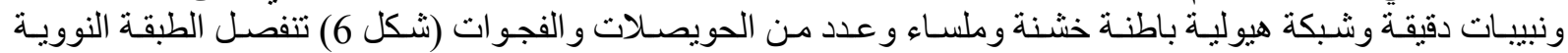

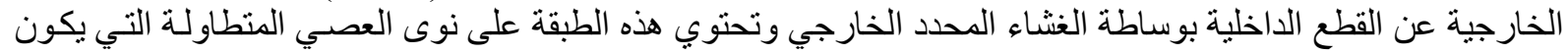

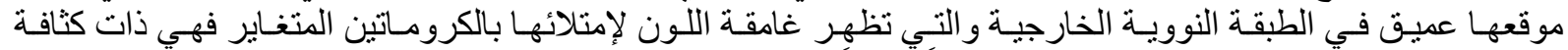

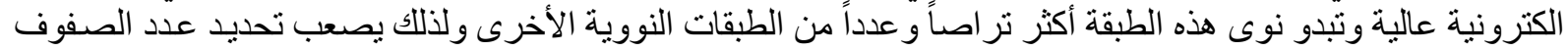

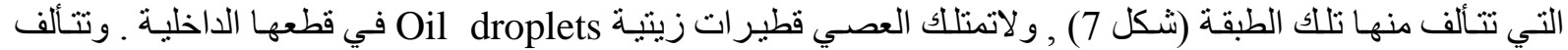

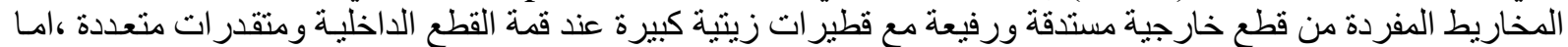

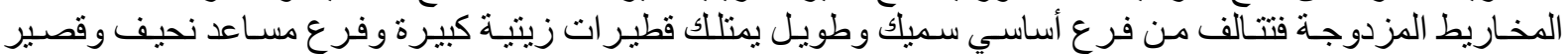

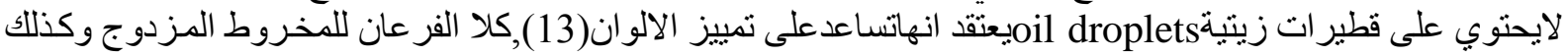

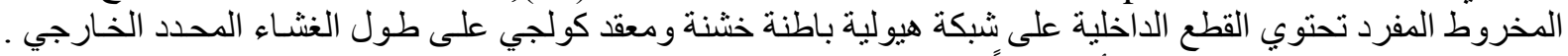

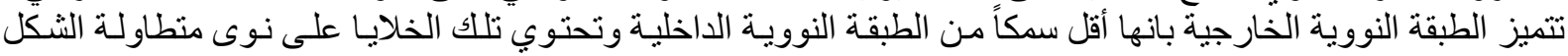

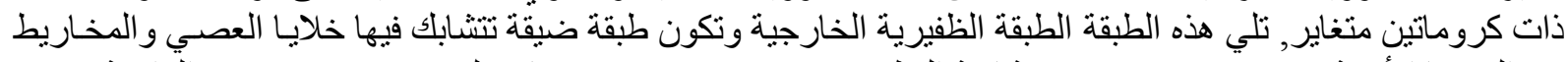

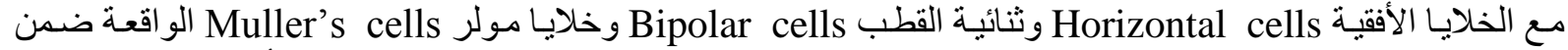

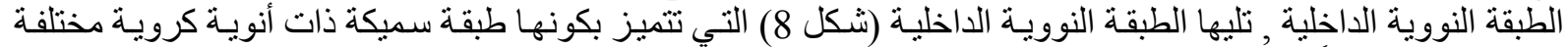

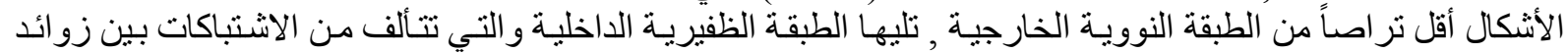

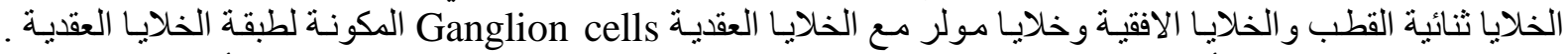

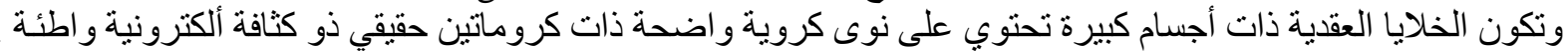

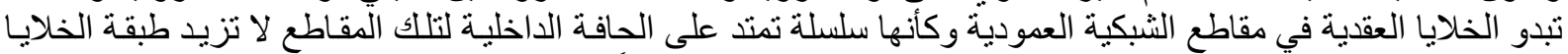

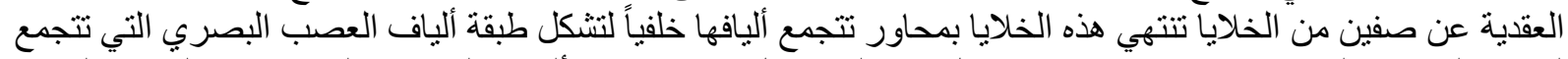

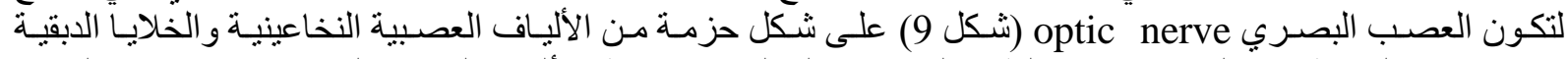
neuroglia

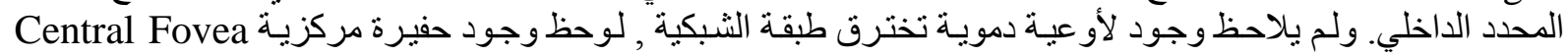


و احدة على سطح الثبكية (شكل 10) وفي بعض أنواع الطيور هناك منطقة او منطقتين في الثبكية تحتوي على مخاريط

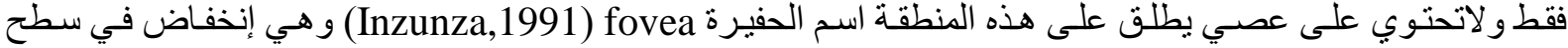

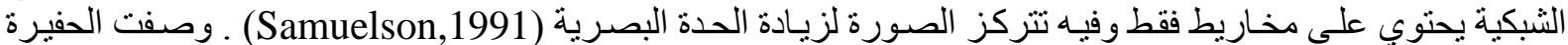

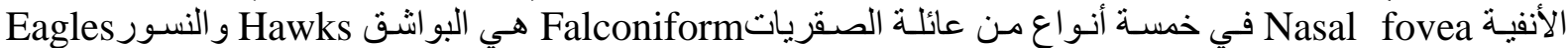

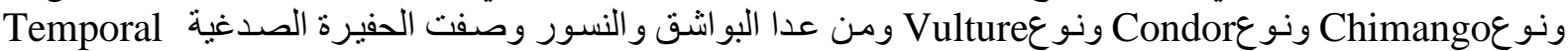
(Inzunza,1993) كذلك fovea
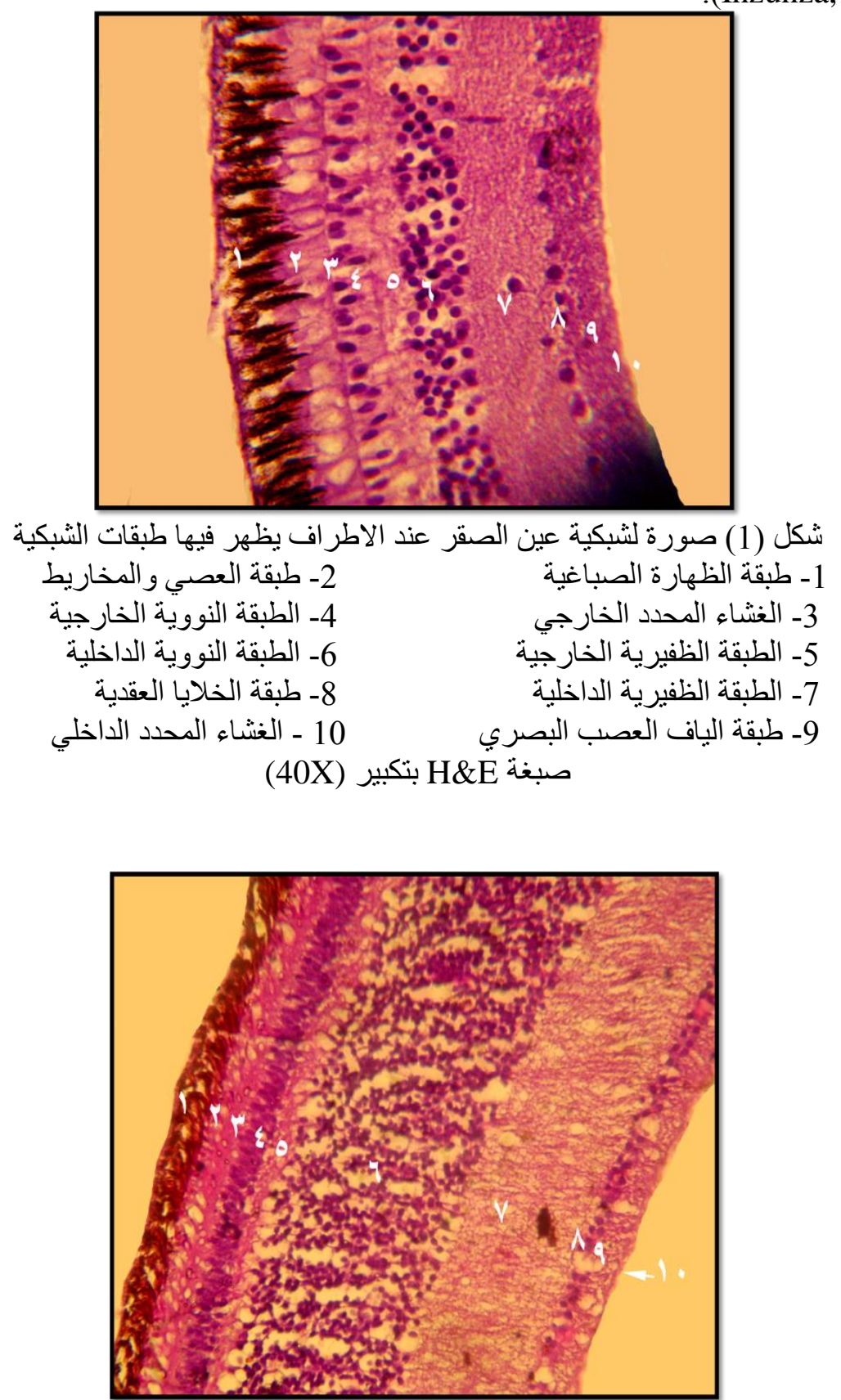

شكل (2) صورة لشبكية عين الصقر عند الوسط لاحظ زيادة سمك الطبقات :

1- Pigment epithelial layer

3- External limiting membrane

5- Outer plexiform layer

7- Inner plexiform layer

9- Optic nerve fibers layer
2- Rod and cones layer

4- Outer nuclear layer

6- Inner nuclear layer

8- Ganglion cells layer

10- Inner limiting membran صبغة H\&E بتكبير (40X) 


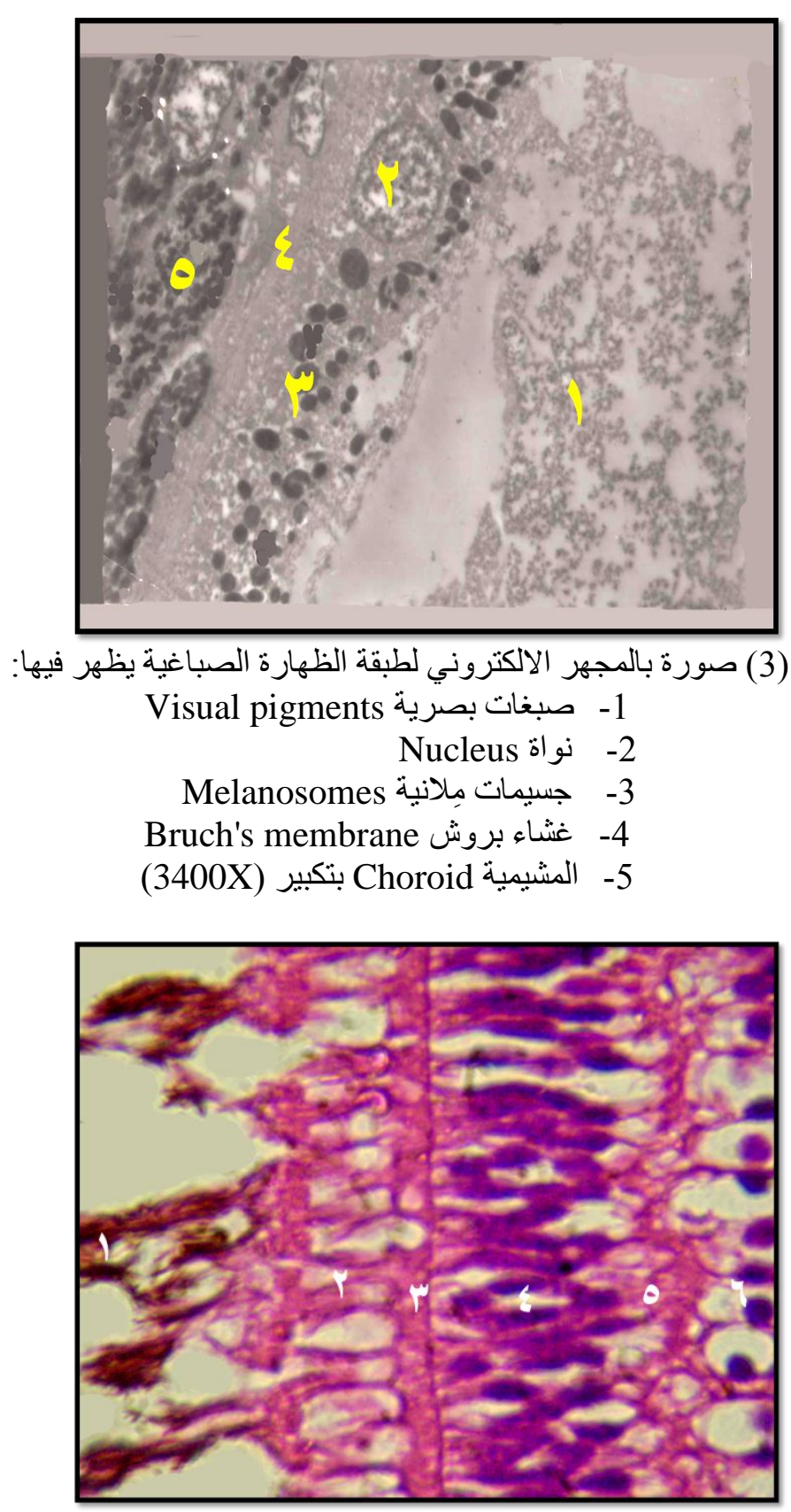

شكل (4) صورة مكبرة للطبقات الامامبة للشبكية يظهر فيها :

1- طبقة الظهارة الصباغية Pigmented epithelial layer

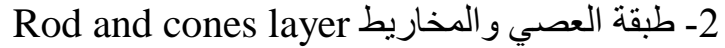

3- الغشاء المحدد الخارجي External limiting membrane

4- الطبقة النووية الخارجية الطية

5- الطبقة الظفيرية الخارجية Outer plexiform layer

6- الطبقة النووية الداخلية Inner nuclear layer صبغة H\& بتكبير (25X) 

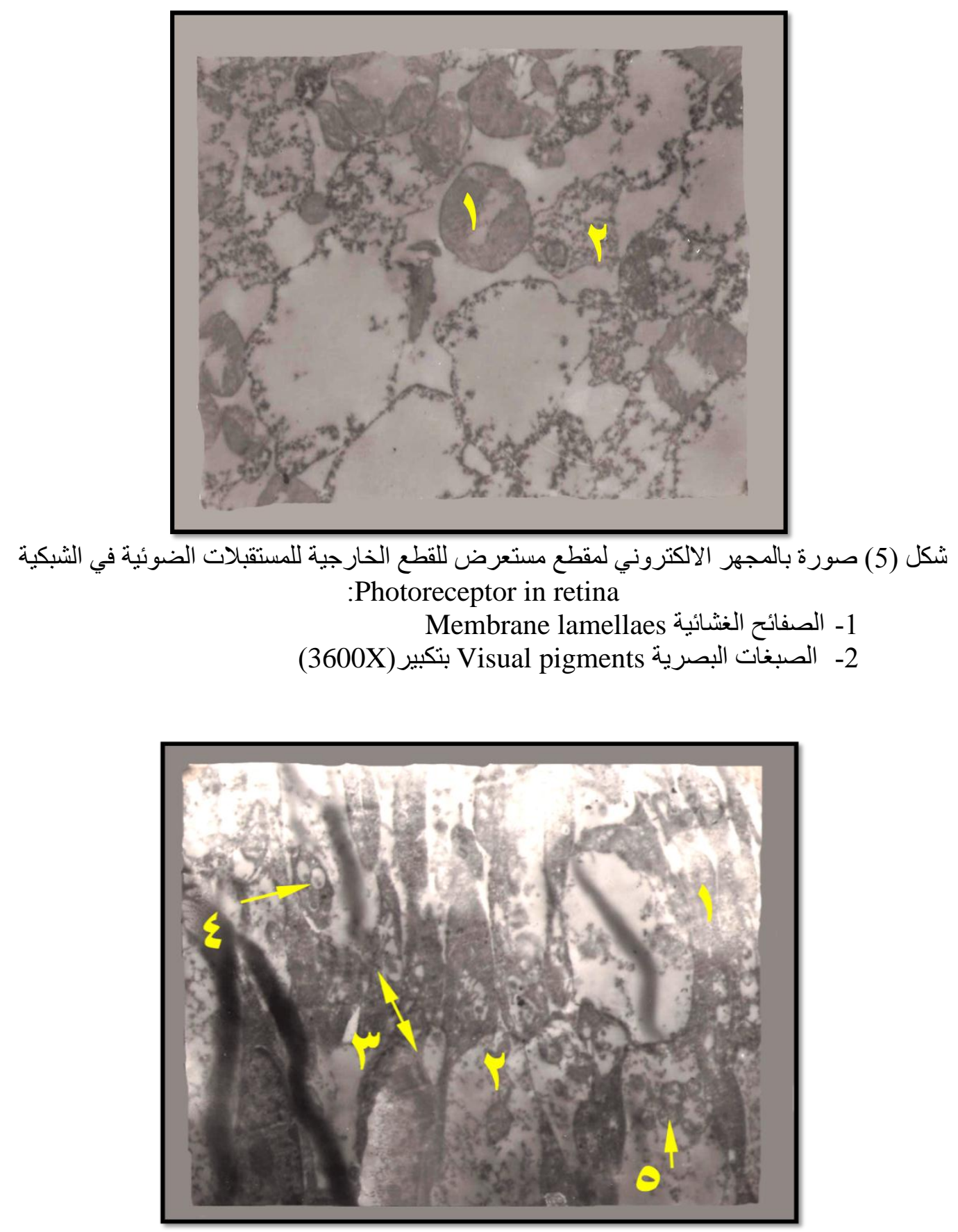

شكل (6) صورة بالمجر الالكتروني لطبقة المستقبلات الضوئية للشبكية

:Photoreceptor layer

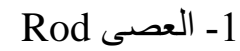

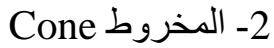

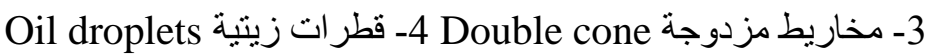

5- المتقدرات Mitochondria بتكبير (2600X) 


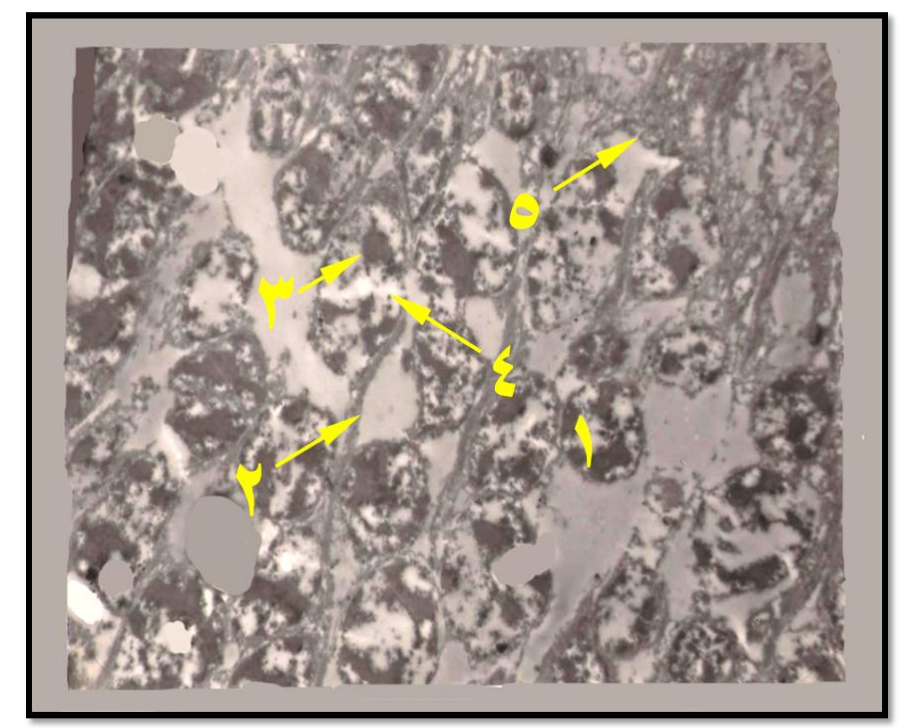

شكل (7) صورة بالمجهر الالكتروني للطبقة النووية الخارجية للشبكية

:Outer nuclear layer

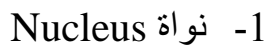

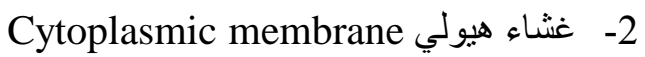

3- كروماتين متغاير Steterochromatin

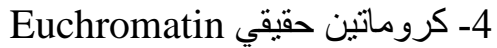

5- غشاء محدد خارجي External limiting membrane

(3600X) بتكبير

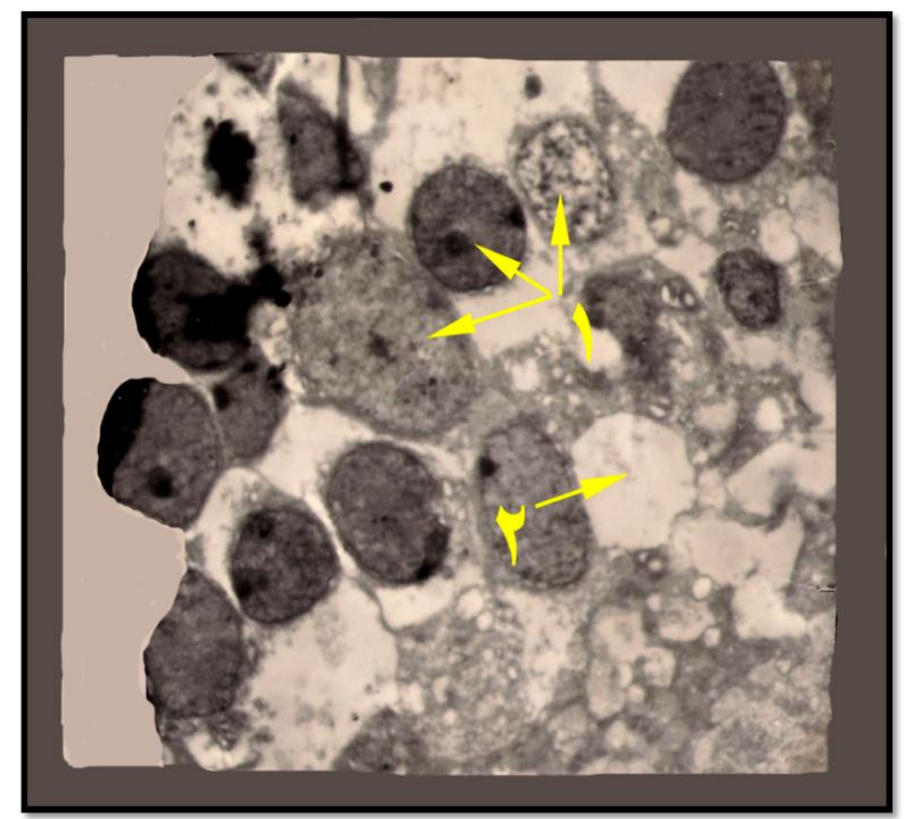

شكل (8) صورة بالمجهر الالكتروني للطبقة النووية الداخلية للشبكية:

1- أنواع مختلفة من النوى Different type of Nuclei النورة

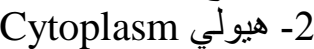

بتكبير (2600X) 

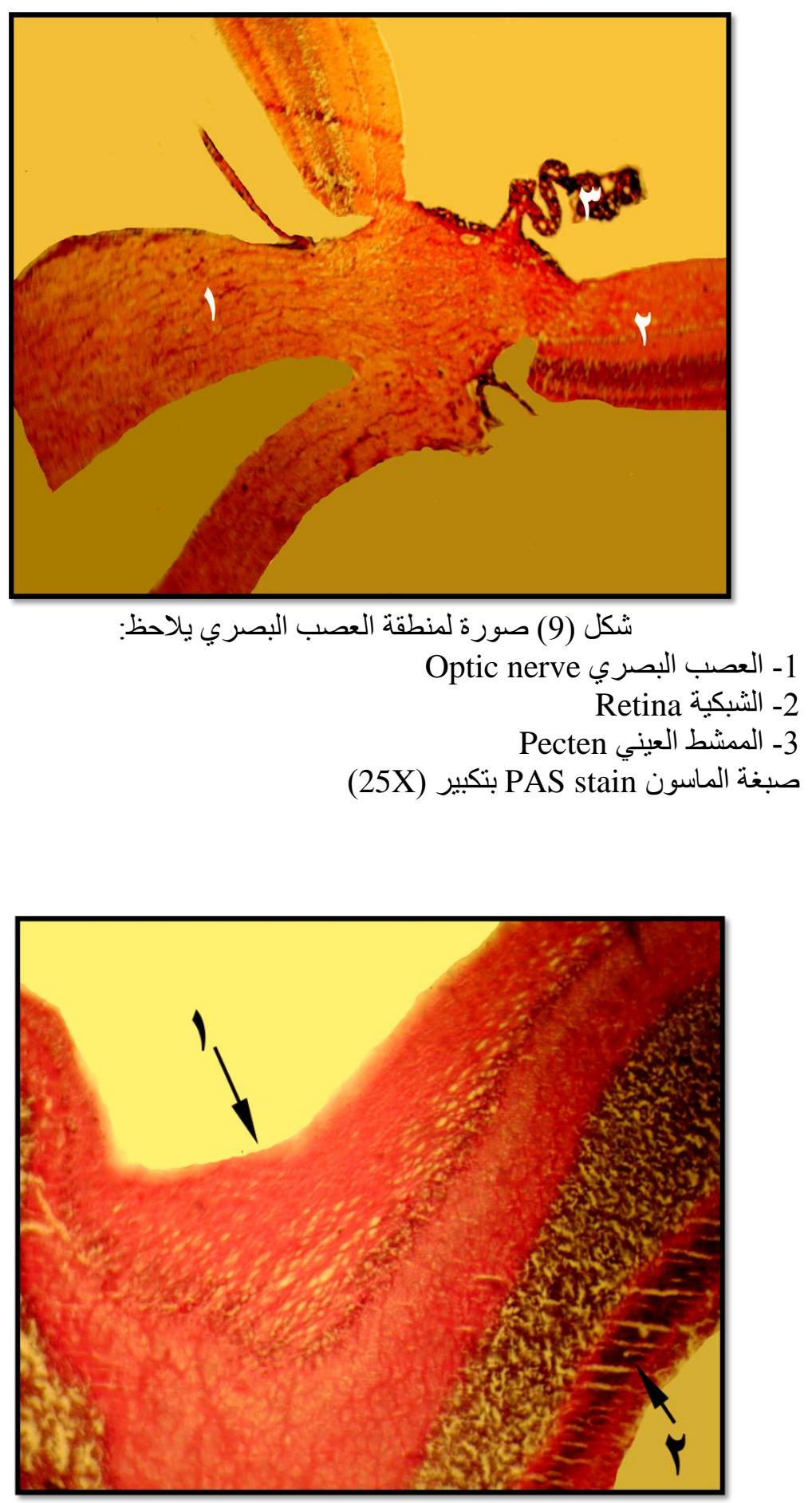

شكل (10) صورة لمنطقة الحفيرة المركزية Central fovea في الثبكية:

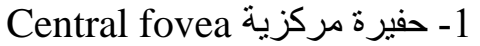

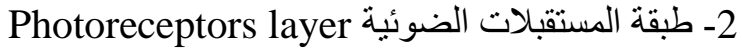

(40x) بنغة PAS بنكبير

\section{المصـــادر}

1. Altunay, H. (2004). Fin structure of the retinal pigment epithelium ,Bruch's membrane and chorio capillaries in the Ostrich (Struthio camelus). Anat. Histol. Embryol. 33 (1): 38 - 41.

2. Bacha, WJ. and Bacha, LM. (2000). Color Atlas of Veterinary Histology. $2^{\text {nd }}$ Ed. Lippincott Williams and Wilkins. P :249. 
3. Braekevelt, CR . (1991). Fine structure of the pectin oculi of the red-tailed hawk (Buteo jamaicensis). Anat. Histol. Embryol., 20: 354-362.

4. Braekevelt, CR. (1994). Retinal photoreceptor fine structure in the American crow (corvus brachyrhynchos). Anat. Histol. Embryol., 23:376-387.

5. Braekevelt, CR. (1998). Fine Structure of the Retinal photoreceptors of the emo(Dromaius novaehollandiae). Tissue and cell, 30: 137-148.

6. Braeckvelt, CR. ; Smith, SA. and Smith, BJ. (1996). Retinal photo receptor fine structre in the Australian Galah (Eolophus roseicapillus ) (Aves). Histol. Histopathol., 11: $555-564$.

7. Braekevelt, C.R. (1993). Fine structure of the retinal photoreceptors of the great horned owl (Bubo virginianus). Histol. Histopathol., 8 (1): 25-34.

8. Mariani , AP. and Leure du pree, AE. (1978). Photoreceptors and oil droplet colors in the red area of the pigeon retina. J . Comp. Neurol., 182: 821 -838.

9. Gondo, M. and Ando, H. (1995). Comparative Histophysiological Study of Oil droplets in the avian retina. Jap. J. Ornithol., 44:81- 91.

10. Goldsmith, TH. ; Collins, JS. and Licht, S. (1984). The cone oil droplets of avian retinas . Vision Res., 24 (11):1661- 1667.

11. Inzunza , O. and Bravo, H. (1993). Foveal topography in the optic nerve and primary visual centers in Falconiforms . Anat. Rec., 235: 623- 631.

12. Samuelson, D. (1991). Ophthalmic anatomy, in Gelatt KN (ed): Veterinary Ophthalmology $3^{\text {rd }}$ ed. Baltimore, MD, Lippincott Williams and Wilkins. Pp: 31-15.

13. Luna, LG . (1968). Manual of histologic staining method of Armed Forces institute of pathology . $3^{\text {rd }}$ ed .Mc Graw - Hill book Company. New York.

14. Preece, A. (1959). Amanual for histologic technics. J. and A. churchill LTD,1st. Ed., London, great Britain.

15. Vacca, L. (1985). Laboratory manual of histochemistry, Ravan press, $1^{\text {st }}$ Ed., New York, U.S.A.

16. Hayat, MA. (1986). Principles and Techniques of Electron Microscopy .Biological Application $.3^{\text {rd }}$ Ed. Macmillan press. P: 405.

17. Aughey, E. and Frye, F.(2010).Comparative veterinary histology. Mason publishing. England, p: 336. 